\title{
Linx
}

Revue des linguistes de l'université Paris X Nanterre

$79 \mid 2019$

Enseigner et apprendre à interagir en langue étrangère : réflexions linguistiques et didactiques

\section{Emprunter sans être impacté ? De quelques effets induits par l'analyse des interactions sur les postures de jeunes enseignants de Français Langue Étrangère}

Borrowing with or without impact? Effects induced by interaction analysis on the stance of early career teachers of French as a Foreign Language

Florence MOURLHON-DALLIES

\section{OpenEdition}

\section{Édition électronique}

URL : http://journals.openedition.org/linx/3671

DOI : 10.4000/linx.3671

ISSN : 2118-9692

Éditeur

Presses universitaires de Paris Nanterre

Référence électronique

Florence MOURLHON-DALLIES, «Emprunter sans être impacté ? De quelques effets induits par I'analyse des interactions sur les postures de jeunes enseignants de Français Langue Étrangère », Linx [En ligne], 79 | 2019, document 6, mis en ligne le 30 décembre 2019, consulté le 07 avril 2020. URL : http://journals.openedition.org/linx/3671 ; DOl : https://doi.org/10.4000/linx.3671

Ce document a été généré automatiquement le 7 avril 2020.

Département de Sciences du langage, Université Paris Ouest 


\section{Emprunter sans être impacté ? De quelques effets induits par l'analyse des interactions sur les postures de jeunes enseignants de Français Langue Étrangère}

Borrowing with or without impact? Effects induced by interaction analysis on the stance of early career teachers of French as a Foreign Language

Florence MOURLHON-DALLIES

\section{L'intégration des caractéristiques de l'oral dans l'enseignement du français langue étrangère : un chemin laborieux}

1 En 2006, C. Weber publiait un article au titre révélateur «Pourquoi les Français ne parlent-ils pas comme je l'ai appris?» soulignant le décalage entre les interactions présentées dans le matériel et les cours de français langue étrangère (désormais FLE) d'une part, et la réalité quotidienne des pratiques langagières dans le monde francophone, d'autre part.

2 Il y a cinq ans environ, quand nous avons pris en charge le cours «Interactions, cultures, apprentissages » de master 2 de didactique à Paris Descartes, nous avons émis une première hypothèse, selon laquelle un tel écart tenait peut-être à un phénomène extérieur à la didactique des langues. Comme le rappelle Kerbrat Orecchioni 2011 : 9 dans son ouvrage bilan Le discours en interaction, la recherche française a quelque peu tardé à se pencher sur les interactions de tous types :

« Il a fallu attendre la fin des années 1960 aux Etats-Unis, et en France, les années

1980, pour que la conversation soit jugée digne d'accéder, au-delà des observations 
« superficielles » dont elle avait dû jusqu'alors se contenter, à la dignité d'un objet scientifique. ».

3 Au vu de ce « retard à l'allumage » (toujours selon Kerbrat-Orecchioni 2011: 11) on aurait pu comprendre que la didactique du français langue étrangère, souvent qualifiée de discipline d'emprunt, ait elle aussi peiné à intégrer la dimension de l'oralité dans le matériel édité. Tout laissait penser cependant que ce décalage allait progressivement s'estomper.

Malheureusement, en 2019 la situation apparaît inchangée en dépit des publications récentes mentionnées dans notre résumé, qui s'attachent à réinvestir les résultats de l'analyse interactionnelle en direction de la classe de FLE ou qui visent la formation des enseignants de langue. Ces publications se sont pourtant multipliées à bon rythme, entre 2010 et 2019.

5 Lassée d'attendre la métamorphose du matériel et des pratiques didactiques, depuis deux ou trois ans, nous en sommes venue à identifier d'autres éléments pouvant expliquer cet écart entre l'oral figuré dans les séquences d'enseignement du FLE et les interactions effectives de la vie quotidienne. Peu à peu, l'emprunt des résultats d'un champ disciplinaire par un autre et la capacité d'un domaine comme la didactique du FLE à accueillir d'autres modes de pensée et de structuration que ceux qui y prédominent, se sont imposés comme sujets de réflexion, par une sorte de choc en retour de l'intelligence collective des générations de mastérants que nous formions dans l'idée de les voir faire fructifier les avancées de l'analyse interactionnelle dans leurs cours de FLE. Il est ainsi apparu au fil des ans que tout emprunt avait un impact, au sens où on n'en ressortait pas indemne, ni comme théoricien ni comme praticien.

\section{I.1 Un matériel édité très limité}

6 Le début de la présente contribution s'attache à montrer que le fossé entre l'oral figuré dans les séquences d'enseignement académique formel et les interactions effectives de la vie quotidienne ne s'est jamais véritablement comblé en presque quarante ans. Dans notre cours de Master 2, nous avons pris le parti d'inviter nos étudiants à identifier et à répertorier les rares îlots conversationnels proches des échanges effectifs que l'on trouve dans le matériel édité, sur la base d'un ensemble d'ouvrages pédagogiques de FLE de tous niveaux (listés en Annexe 1). Par une telle cartographie, on espérait avant tout rendre les étudiants futurs enseignants de FLE conscients des limites de ce qui est proposé par les éditeurs les plus influents.

\section{I.1.1 Tour d'horizon de la production éditoriale}

7 Toutes périodes confondues, l'analyse du matériel édité en France pour le FLE révèle une très faible appétence en matière d'écoute et de reproduction d'interactions authentiques ou même simplement « réalistes ${ }^{1}$. Une analyse (qualitative et non exhaustive) du matériel à large tirage produit par les principaux éditeurs de FLE ces trente dernières années fait rapidement apparaître que les ouvrages qui se réclament ouvertement d'un travail du français oral ou accordent une place conséquente à la sensibilisation aux traits d'oralité relèvent de la catégorie des auxiliaires pédagogiques et non des manuels eux-mêmes. 
On pense notamment aux deux publications de Lebre-Peytard A l'écoute des Français en 1991 puis A l'écoute des professionnels en 1994, chez CLE International, qui fonctionnent comme des cahiers d'activité. Egalement à part des manuels, il existe aussi le livret accompagné de vidéos Clés pour l'oral (Hachette FLE 2011) élaboré par Calbris et Montredon. Le dernier cahier spécialisé en date est paru en 2015 chez Hachette FLE : il s'agit de Paroles En Situations, issu d'une collaboration entre Laurens et Guimbretière. Ce titre fait lui-même écho à l'ancien Paroles publié par Guimbretière en 1992 chez Hatier. Le travail du français oral tel qu'il est parlé semble donc cantonné dans le matériel édité à des publications de moins large rayonnement que les manuels, c'est-à-dire à des supports de format plus léger que l'on utilise dans des plages de cours spécifiques, occasionnelles, en dehors du déroulement ordinaire et régulier des enseignements académiques.

Cette segmentation du marché éditorial correspond à la réalité de la plupart des enseignements de FLE dans le monde. Dans certains pays et contextes (comme les lycées et les universités chinoises) les manuels préparent avant tout à des examens écrits, ce qui limite le travail de l'oral à quelques éléments de prononciation, sans que soit développée la mise en pratique de la langue par la production de dialogues ou la prise de parole lors de débats. Dans la plupart des endroits où l'oral est cependant amplement travaillé (notamment par le biais des approches communicative et postcommunicative) l'attention n'est que rarement portée aux caractéristiques du discours en interaction: constructions telles les dislocations, les clivées, les pseudo-clivées, qui permettent de mettre en avant tel ou tel point prêtant à la discussion lors d'un échange amical, familial ou professionnel ; phrases inachevées, qui marquent un embarras, un temps de réflexion, ou passent silencieusement le relais à l'interlocuteur; chevauchements qui montrent un emballement du propos, une dispute, un enthousiasme partagé ; usages de ponctuants, qui rendent visibles le changement de sujet abordé, la clôture du propos; signes de validation interlocutoire marquant discrètement l'intérêt de l'interlocuteur et appelant le locuteur à poursuivre ; pauses liées à la manipulation d'objets ou à l'irruption d'un nouvel interactant dans la conversation. La liste n'est pas exhaustive mais montre la variété des phénomènes qui sont laissés généralement de côté et tiennent autant du verbal que du paraverbal et du non verbal. Quand toutefois ces éléments sont pris en compte, les enseignants n'y consacrent souvent que quelques séances isolées sur l'année, ce qui explique le choix de traiter de ces caractéristiques par le biais de fascicules réduits, à usage souple.

Et encore cela ne garantit pas la vente des cahiers spécialisés en la matière. Ignorant fréquemment l'existence de matériel dédié, les professeurs de FLE se focalisent sur les rares passages des manuels traitant de l'expression de l'émotion au travers de variations d'intonation et d'interjections diverses. Cela est entre autres proposé dans Latitudes 3, p. 127 à l'occasion de l'entretien enregistré d'une jeune Médecin du Monde, qui concentre de nombreux ponctuants de l'oral. Il arrive aussi que certaines méthodes récentes de FLE (souvent associées à de la vidéo ou du numérique) travaillent la combinaison des gestes et des paroles, à l'occasion de salutations ou de requêtes principalement. En pointe sur la question, Texto 1 met en regard des photos et des répliques (dans la rubrique dédiée " Faits et gestes ", comme p.22 ou p. 74). Toutefois, il ne s'agit pas d'un traitement explicite des caractéristiques de l'interaction, mais plutôt d'ouvertures qui permettent à l'enseignant d'aborder la dimension interactionnelle au 
détour d'autres rubriques (l'expression des sentiments ou le travail de l'interculturalité).

11 Il est à noter que les manuels des niveaux les plus élevés (2 et 3) ne traitent pas la dynamique interactionnelle et ses caractéristiques de manière exhaustive. Ce point émerge de manière anecdotique, alors qu'on pourrait s'attendre à ce qu'au fil des ans les subtilités du français oral soient systématiquement abordées. On peut toutefois mentionner l'exception que constitue Reflets (Hachette 2000) qui présentait à l'époque de sa parution des scènes filmées. La méthode offrait également des exercices indiquant comment «Interrompre une conversation », p.14 ou " Changer de sujet », p.56, ce qui en fait l'un des rares outils pédagogiques permettant d'entraîner à la gestion des interactions.

12 Ces quelques « moments de grâce " manifestent que l'oral et l'oralité sont deux choses bien différentes : soumettre des dialogues fabriqués peu représentatifs des interactions quotidiennes revient à faire écouter des documents sonores mais ne garantit pas que les étudiants soient sensibilisés aux caractéristiques des conversations et interactions de tous types. Au vu du nombre de supports enregistrés ou filmés, il ne manque pas en effet d'enregistrements dialogués dans les manuels. Mais on se trouve là devant un paradoxe : alors que les dialogues se multiplient à chaque unité, leur caractère dialogué est presque inexistant dans tout le matériel édité. La plupart du temps, et bien que deux personnes soient censées se parler, on a affaire en réalité à des monologues lancés par une question de journaliste (comme dans Latitudes 3, avec l'interview du maire du Séquestre ", Activité 1, p. 143). Parfois même, une voix off vient couvrir les échanges authentiques, qui ne sont donnés à entendre que très ponctuellement, comme dans le document vidéo sur le repas gastronomique des Français, d'Edito B1 de 2012. Il est surprenant que ce phénomène soit courant dans les manuels de niveau 3, qui auraient pu introduire des tours de parole plus alternés, et plus étonnant encore de voir ce travers bien installé dans le matériel pour adultes professionnels, comme constaté dans Mourlhon-Dallies 2018a.

Loin d'avoir affaire à des polylogues vivants, modelés au gré des allers et venues de personnages actifs et parfois imprévisibles, le dialogue est donc, quel que soit le niveau de langue et la nature du public, limité à quelques questions et réponses saupoudrées de « petits mots » (Traverso 2011 : 45-49). Pour donner l'impression d'un oral vivant et spontané, les auteurs recourent à l'interrogation intonative («Tu viens?») et à quelques points de suspension pour marquer l'hésitation, ce qui induit un «effet d'oralité » comme si on cherchait à suggérer une conversation plutôt que d'en produire une. Un tel résultat issu de l'analyse de manuels produit un choc doublé d'une grande frustration chez les mastérants ${ }^{2}$, car l'écart entre ce qui est actuellement su de la langue parlée en interaction et ce qui en est donné à entendre est immense. Différentes visions $d u$ « français à faire apprendre » sont alors en confrontation : celle de la langue correcte et de ses usages principaux normés souvent scriptocentrés, que véhiculent la plupart des manuels et des enseignants ; celle de nos propres mastérants formés à la linguistique de corpus, qui espèrent mettre à profit les résultats des études de linguistique formelle mais qui n'échappent pas à une certaine tentation applicative ; notre propre intérêt pour l'interdisciplinarité et les emprunts d'une discipline à l'autre; les motivations parfois contradictoires des apprenants, pris entre le désir d'exceller dans un univers académique mais qui espèrent aussi se fondre dans les échanges en pays francophone, lors de séjours occasionnels ou de mobilités longues. Il 
ne nous échappe donc pas que le bilan que nous opérons tient à nos propres intérêts ainsi qu'à la formation reçue en M1 puis en M2 par les cohortes d'étudiants avec lesquels nous avons analysé le matériel.

14 Avec les préoccupations qui sont les nôtres, la période contemporaine est même particulièrement décevante : l'intégration des avancées de l'analyse conversationnelle reste étonnamment timide. Les échanges discontinus, hachés, riches en dislocations, marqués par des bifurcations du propos ou par l'irruption de paroles incidentes (nouveau venu, appel téléphonique) ne sont guère plus représentés dans les manuels que par le passé. Alors que les manuels numériques se développent (tels Alter ego+), ce qui permettrait en théorie d'articuler davantage le verbal au non verbal, les dialogues sont plutôt moins étoffés qu'auparavant dans les nouvelles méthodes; de fait, les maisons d'édition optent toutes pour la multiplication de très brèves scènes plutôt que pour la présentation d'un dialogue d'appui long, qui serait pourtant plus favorable à l'illustration des dynamiques de co-construction du discours à l'œuvre. Cette brièveté caractérise y compris le matériel récent comme Paroles En Situation A2/B1. À l'image de l'épisode de l'évier bouché (p. 81), les extraits dialogués sur le vif sont très brefs, comme distillés au compte-gouttes, et servent d'appui à une transcription de quelques lignes, à partir de laquelle il est demandé de réinstaller des indications d'intonation ou de prononciation. On trouve également dans ce matériel spécialisé, beaucoup de compréhensions fines sur le mode du QCM, selon les souhaits de l'éditeur qui voulait que le matériel puisse être utilisé en autonomie par les étudiants de FLE, sur le mode auto-correctif. Dans ces conditions, la dynamique conversationnelle ne peut pas être complètement approfondie et les étudiants en restent le plus souvent à la compréhension orale et au relevé d'une ou deux caractéristiques interactionnelles alors même que les auteurs du matériel avaient la matière pour aller jusqu'à la production (ce qui transparaît davantage dans l'extension numérique dédiée). Cela étant, la publication d'un auxiliaire pédagogique consacré à l'oral est une prise de risque pour la maison d'édition et l'on comprend que le matériel soit le fruit de compromis.

\subsubsection{Analyse de détail de dialogues fabriqués actuels}

15 Afin de bien comprendre quels sont actuellement ces compromis, nous avons fait le choix de travailler sur des extraits de matériel contemporains. Pour donner une idée des dialogues qui y figurent, nous avons pris pour point de réflexion principal le manuel paru en juin 2019 chez Maison des Langues A la Une: au cour du monde francophone qui se réclame du courant actionnel. On tient là, à la date de rédaction qui est la nôtre, le manuel le plus récent que l'on puisse espérer. Prenons l'exemple du dialogue enregistré de l'unité 7 (piste 41), qui consiste en un échange téléphonique du tac au tac. Ce dernier intègre différents traits d'oralité (comme des interrogations intonatives, des expressions typiques telles "ça marche" et l'insertion d'un OK). Toutefois l'ensemble demeure encore assez peu naturel :

- Tu es où Line? Je suis à l'arrêt de métro Vieux Lyon.

- Je suis tout près, j'arrive!

- Mais tu es où exactement?

- Je suis au palais Saint-Jean, juste derrière l'avenue Adolphe-Max.

- Ok, on va à Confluence après?

- Ça marche ! On y va à pied?

- Non, je préfère aller en tram ! 
seconde étape d'analyse permet d'objectiver à quoi attribuer le caractère partiellement artificiel perçu à la lecture de ce dialogue de manuel. On remarque ainsi que chaque réplique y prend la forme d'une ou deux phrase(s) syntaxiquement complète(s), sans que la réplique suivante ne la chevauche ni ne la prolonge, ce qui n'est pas conforme à l'enchaînement habituel du français oral. La fin du paragraphe propose en particulier une réponse de type sujet verbe complément « je préfère aller en tram » qui aurait pu être avantageusement remplacée par « Non, en tram... je préfère » ou « Non en tram plutôt! » selon la logique conversationnelle qui veut qu'on se focalise sur l'élément de la négociation verbale en cours (ici, le moyen de transport). Cet élément est généralement mis en premier dans la réponse orale à une question, en français quotidien. De même la première réplique «Je suis à l'arrêt de métro Vieux Lyon " serait, dans une conversation courante réduite à "Moi, je suis à Vieux Lyon » (indication éventuellement suivie de « ... dans le métro »). Un peu plus loin, la précision "je suis au palais Saint-Jean » donnerait plutôt l'enchaînement « Moi, au palais Saint Jean, tu sais, juste derrière l'avenue Adolphe-Max ». L'ajout du « moi » permettrait de souligner l'opération de localisation et renverrait plus fidèlement à l'activité de coconstruction du point de rendez-vous. Enfin l'insertion d'un « tu sais » rendrait plus net l'engagement des deux personnages dans l'échange téléphonique en cours, en jouant le rôle de "marqueur phatique " portant la continuité du discours et "appelant une manifestation d'attention de la part de l'interlocuteur » (Traverso 2011 : 47).

À l'image de ce que nous venons de faire pour un manuel paru en juin 2019, soit après notre période de cours en master 2 (nous enseignons en effet au semestre 1), nous avons conduit sur des manuels plus anciens ce type de démarche avec nos étudiants, laquelle consiste à décrire précisément les traits d'oralité dont les dialogues de manuels sont parsemés dans une première analyse puis à identifier dans un second temps, par une relecture attentive, les éléments qui auraient pu s'y rencontrer compte-tenu du contexte, de la tonalité et des visées de l'échange figuré, mais qui ne s'y trouvent pas. Comme nous nous attachons à le montrer tout au long de la présente contribution, on est aujourd'hui en mesure de conduire une analyse dégagée des simples intuitions. Des catégories descriptives particulièrement fines ont en effet été mises en circulation dans le champ de l'analyse conversationnelle et interactionnelle depuis une petite dizaine d'années, si bien qu'il est aisé d'objectiver ce qui autrefois n'aurait été qu'une impression fugace d'absence de naturel.

Pour rendre justice au matériel pédagogique actuel, tout n'y est pas cependant «artificiel ». Certains progrès, certes très ponctuels, ont été accomplis. Ainsi A la Une, dont nous avons vu les limites plus haut, témoigne par endroits d'une intégration de la dynamique $d u$ français en interaction plus importante que ses prédécesseurs. La multiplication des marqueurs interactionnels brefs (ou "petits mots ", selon Traverso 2011) les reprises d'un interlocuteur à l'autre, les enchaînements où ce qui est dit par un personnage est complété par le suivant, donnent lieu par moments à des passages très crédibles. C'est le cas de l'unité 6 piste 36, relative à la planification d'un week-end entre jeunes. Les répliques des interlocuteurs se font écho («Pas de problème! Et après on va faire de la voile?/ De la voile? Tu rêves ! Je déteste l'eau !») ou se complètent (Concert de qui ?/Odezenne à $19 \mathrm{~h} 30$ sur les quais! Moi j'y vais avec Arthur et Yannis). La clôture aussi est, dans ce dialogue, plus complexe que la moyenne, avec l'expression d'une approbation, l'annonce d'un rappel ultérieur très probable et le cumul 
d'expressions typiques de l'oral (Bonne idée ! On se redit alors !/Super, ciao !/Bye) dont une partie empruntée à d'autres langues, très présentes dans le parler quotidien.

Ce travail d'analyse de manuels récents a également été conduit pour les manuels de français professionnel. Nous avons établi dans Mourlhon-Dallies 2018a une liste de caractéristiques des interactions au travail, qui montre combien les objets et les manipulations de tous types structurent celles-ci ${ }^{3}$. Or, dans les manuels de spécialité, les dialogues demeurent encore très désincarnés, selon le rapide tour d'horizon du matériel édité déjà esquissé dans Mourlhon-Dallies 2018, b. Sur la base d'un sous-corpus de manuels de français professionnel (Annexe 1, titres suivis de l'astérisque), on a pu constater que les parutions en français de spécialité les plus récentes n'étaient pas forcément les plus représentatives des interactions effectives. À l'image du Français en contexte tourisme 2016, les enregistrements proposés y sont soit des témoignages longs de professionnels (quasiment sans alternance de tours) soit des échanges d'une ou deux minutes dont des conversations téléphoniques entre clients et employés, fabriquées et prises «en cours de route», sans leur ouverture ou sans clôture, ou alors avec des séquences liminaires minimales.

Ainsi l'intégration des caractéristiques désormais bien connues des interactions reste encore très partielle dans les manuels de français professionnel comme de français général ${ }^{4}$. On est donc amenée à conclure que la majorité du matériel actuel ne va guère plus loin que ce qui était déjà amorcé dès les années 2000, ce qui amène à s'interroger sur les raisons de cette situation.

\section{I.2 Tentatives d'explication}

21 La faible intégration au matériel pédagogique de FLE des résultats de recherche issus de l'analyse conversationnelle et interactionnelle ne peut pas être imputée, selon nous, à une ignorance des caractéristiques de l'oral de la part des didacticiens. «Les traits de l'oralité » sont effet connus depuis longtemps de ces derniers comme en témoigne la liste établie par Lebre-Peytard et Malandain 1982: 29. Certains de ces points ont fait l'objet de développements didactiques spécifiques comme dans Lhote 2001, avec Enseigner l'oral en interaction, Percevoir, écouter, comprendre centré sur la dimension mélodique et intonative des échanges verbaux. Plus proche dans le temps, Weber 2013 a établi dans Pour une didactique de l'oralité. Enseigner le français tel qu'il est parlé, un récapitulatif très complet de tout ce qui peut faire l'objet d'un travail de l'oral en classe de langue. Un schéma (p. 262) synthétise sous la notion de «charpente de l'oralité » le foisonnement des éléments structurels et communicationnels qu'il est possible de prendre en compte.

On voit là que des enseignants chercheurs pleinement inscrits dans le champ de la didactique du FLE n'ignorent en rien les éléments descriptifs clés du français oral en interaction. Le titre de Weber 2013 sonne néanmoins comme un manifeste, en commençant par un «Pour » qui signale que les habitus didactiques ne sont pas encore en phase avec ce que la connaissance bâtie en la matière par les différentes branches des Sciences du langage permettrait de mettre en œuvre. Mais comment expliquer en 2019 une telle frilosité en matière d'insertion des traits d'oralité et la prise en compte si parcimonieuse des dynamiques conversationnelles dans les manuels et dans les auxiliaires pédagogiques de tous types? 


\section{I.2.1 La prégnance de l'écrit} transcription du document oral brut en classe de français ; ils indiquent ce faisant que lors de l'opération de restitution mot pour mot d'un enregistrement audio on bascule facilement vers une mise en conformité avec l'écrit, qui peut conduire, sans même parler de remodelage d'un dialogue à des fins pédagogiques, à une version expurgée des principaux traits d'oralité. En effet,

«La première réaction à la transcription d'un document sonore sera de dire que « ce n'est pas du français ». Par la suppression des hésitations, des redondances, des répétitions, des éléments phatiques [...] et par l'emploi de la ponctuation qui segmente l'énoncé en groupes de sens on tend, consciemment ou non, à retrouver l'organisation et la «norme» du texte écrit ». (Lebre-Peytard et al. 1977, p. 5, fascicule dactylographié).

Par-delà ce réflexe d'un retour au code écrit, se pose aussi très certainement la question de l'acceptabilité des caractéristiques de l'oral face à l'image normée de la langue, aux yeux des institutions éducatives et des enseignants eux-mêmes. Rivenc explique bien des années après la réalisation du manuel Voix et Images de France avoir supprimé des enregistrements authentiques qui lui servaient d'appui quasiment tout ce qui ne relevait pas d'une grammaire de l'écrit standard. De son propre aveu, les esprits n'étaient pas prêts: "Les modèles de référence restaient bien, secrètement mais fermement, ceux de la langue écrite " (Rivenc 2006: 6). Ces confidences tardives montrent qu'en matière de conception de matériel, l'idée que se font les auteurs de manuels de la marge de manœuvre dont ils disposent pour présenter à des apprenants la langue française orale et ses usages serait à explorer systématiquement ; «l'espace d'exposition discursive» (Develotte 1995) que chacun s'autorise à proposer aux étudiants avec plus ou moins d'audace est très certainement une notion à réactiver.

\section{I.2.2 Le poids des méthodologies didactiques}

Un autre facteur explicatif, propre au champ disciplinaire de la didactique des langues, peut également être avancé pour comprendre la rareté des traits d'oralité dans le matériel édité. Porquier $2001: 119$ suggère que la prédominance depuis les années 1980 de l'approche communicative est susceptible d'avoir joué contre l'intégration systématique et massive de certaines caractéristiques de l'oral : le peu de place laissée aux « locutions énoncés »,

« tiendrait à la difficulté apparente d'élaborer des activités ou des exercices spécifiques pour l'apprentissage d'unités disparates, sans rapport avec les activités instituées et routinisées pour l'apprentissage de la grammaire et du lexique. L'entraînement à l'emploi de au secours, de attention, de ça y est se prête mal à des exercices systématiques, mais plutôt, à des niveaux avancés, à des activités inductives d'observation et de réflexion sur les valeurs d'emploi de telles unités ». (Porquier 2011 : 119).

Traiter ou pas tel ou tel point de langue dans les manuels renverrait donc aussi aux notions et aux pratiques didactiques qui en assurent la structuration. À cela s'ajoute, selon nous, la logique même d'exploitation des échanges conversationnels, telle qu'elle a été historiquement façonnée par les didacticiens pionniers dans le domaine. Le choix de soumettre à l'écoute de brefs extraits a en effet été posé très tôt par Lebre et al. 
1977 : 17-19 dans le chapitre intitulé «Utilisation pédagogique de la transcription ». Mais en considérant que l'oral pouvait se travailler à partir d'échantillons présentés occasionnellement aux étudiants pour opérer une sensibilisation à ses spécificités, les rares moments de cours consacrés à l'exposition des étudiants à des interactions authentiques ont été faits essentiellement sur le mode de la réception et non sur celui de la production. Le fait de s'en tenir le plus souvent à une familiarisation ou à une prise de conscience a d'une certaine manière limité la manuélisation des catégories descriptives de l'analyse interactionnelle et a réduit le travail de systématisation en cours de langue. Il faut souligner enfin, qu'en dehors de l'analyse d'extraits, les échantillons utilisés à des fins pédagogiques dans le matériel édité ont surtout été soumis à des compréhensions plus ou moins fines, comme préconisé dès les années soixante-dix. Mais là encore, qui dit compréhension ne dit pas production ni réinvestissement des caractéristiques auxquelles les étudiants sont sensibilisés.

Prétextes à un travail interprétatif ponctuel, les avancées de l'analyse interactionnelle n'ont donc pas été intégrées de manière massive aux activités de production orale, qui passent généralement dans un cours de FLE par des jeux de rôles fondés sur l'imitation de dialogues fabriqués, peu représentatifs des échanges oraux spontanés. Et cette manière d'exploiter les extraits authentiques ou quasi-authentiques principalement par l'écoute et la compréhension perdure, si bien que la production orale est le parent pauvre de la didactisation des interactions verbales « naturelles » conduite en classe de FLE, en contexte post-communicatif. Et si l'on aborde l'approche actionnelle, qui s'impose peu à peu comme méthodologie dominante, cette dernière pourrait être moins favorable à la présence de dialogues longs que le communicatif. L'actionnel pousse en effet les étudiants à s'exprimer en tant que personnes plutôt qu'à reproduire des avis ou des scènes préalables. De fait, pour travailler les émotions, au lieu de se limiter à faire décoder si tel ou tel personnage est triste ou indigné, un manuel comme Adomania, A1/A2 demande (Leçon 2, p. 40) également et surtout aux étudiants de dire dans quelles circonstances ils sont heureux, excédés ou honteux, ce qui accentue le témoignage, plus monologal.

\section{Des corpus numériques « compensatoires »}

Alors que les manuels peinent à présenter des échantillons de langue parlée et que les résultats de la recherche sur les interactions ne font pas l'objet d'une appropriation systématique dans le matériel édité pourtant dédié à l'oral, une solution pourrait bien être de se tourner vers les corpus numériques d'interactions récemment mis à disposition du grand public et des didacticiens et apprenants de langues. Depuis une trentaine d'années de nombreux corpus oraux numérisés, dont certains sont présentés dans ce numéro de Linx, ont en effet vu le jour. On peut retrouver les principaux d'entre eux sur le portail cOCOON (https://cocoon.huma-num.fr/exist/crdo/) ou encore sur la plate-forme Orféo (https://www.projet-orfeo.fr/corpus-source/) mais chacun dispose bien entendu de son site propre. Les plus connus des enseignants de FLE sont répertoriés en Annexe 2. Dans le cours «Interactions, cultures et apprentissages » une séance entière est consacrée à la découverte de ces ressources clés, en salle informatique. L'analyse de ces sites est ensuite poursuivie méthodiquement, en dehors des plages d'enseignement universitaire en face à face, avec un suivi en ligne. 


\section{II.1 Des corpus convertis aux préoccupations didactiques, comme " après coup "} du FLE était menée dans notre cours de master 2 essentiellement dans le but d'informer
les professeurs de FLE en formation initiale de l'existence de ressources gratuites pouvant compléter de temps à autre les manuels quand on voulait présenter en cours de l'oral « authentique ». Cela montre que nous avons considéré initialement les corpus oraux numériques comme périphériques - avant d'en faire des instruments d'interrogation privilégiés de la discipline "didactique des langues». Ce n'est donc qu'au fil des ans que l'analyse des corpus numériques d'interactions est devenue en cours l'occasion de nombreux échanges beaucoup plus formatifs.

\section{II.1.1 Des projets de nature linguistique, à l'origine}

Une partie non négligeable des discussions avec les mastérants porte sur les conditions d'élaboration des sites de corpus oraux, car critiquer l'existant reste peu intéressant de notre point de vue si on ne forme pas les futurs utilisateurs de ces collections numérisées et souvent prépédagogisées à débattre des choix opérés par leurs auteurs. À cet égard, un point revient très souvent dans les échanges, à savoir la faible implication des didacticiens du FLE dans la création de ces sites. On voit là que mettre à nu les conditions d'élaboration de matériel didactique n'est pas neutre au plan institutionnel. Les étudiants n'interrogent pas seulement les limites des sites numérisés : ils entrent dans l'histoire complexe des relations entre linguistique et didactique des langues, en se posant des questions d'indépendance disciplinaire, de places dominantes ou périphériques dans les réseaux académiques, mais aussi d'épistémologie de l'interdisciplinarité. Cette prise de conscience peut avoir l'allure d'une déflagration car une fois la posture réflexive adoptée, surgissent aussi d'autres objets à interroger (comme les prises de parole durant les cours de l'enseignant de langue lui-même, la nature des épreuves orales des certifications de niveau de langue); tout cela à la manière d'une réaction en chaîne.

Si on s'en tient à l'analyse des corpus de langue parlée numérisés, il apparait très rapidement qu'en dehors du projet ESLO de «français orléanais » qui «à la fin des années soixante autour de Michel Blanc avait comme objectif de réaliser une méthode d'enseignement audiovisuelle du français» (Baude et Dugua 2016), les initiatives de constitution de collections de français parlé avaient, à leur origine, une vocation autre que didactique. Projets "patrimoniaux» ou bien soutien à des programmes d'étude linguistique et descriptive $\mathrm{du}$ français oral, les corpus de grande ampleur ambitionnaient de servir d'appui à des travaux relevant des Sciences du langage et occultaient très souvent les retombées possibles en didactique des langues. Il reste à cet égard frappant que le chapitre 2.1.5 du Guide de bonnes pratiques produit par Baude et al. 2006 et consacré aux exploitations des corpus oraux ne mentionne pas explicitement la didactique des langues comme discipline bénéficiaire de ces bases de données alors que la fabrication de grammaires des langues nationales, l'amélioration des connaissances de la grammaire en interaction, le traitement automatique de la parole et la comparaison des langues entre elles sont citées comme champs scientifiques directement intéressés par les activités de collationnement des grands corpus numériques. 
L'exploitation dans l'optique FLE des vastes corpus oraux n'a donc pas été et n'est toujours pas une évidence dans le milieu académique de recherche français. Et même des sites aujourd'hui entièrement dédiés à l'enseignement du FLE, tel CLAPI.FLE (http://clapi.ish-lyon.cnrs.fr/FLE/liste_extraits.php) ne se sont imposés qu' "après coup » dans les projets de recherche dont ils relèvent. CLAPI-FLE est en effet un produit dérivé relativement récent (et encore en cours de constitution) d'un site antérieur nommé CLAPI. Comme précisé dans le présent numéro de Linx par Ravazzolo et Etienne mais aussi dans un bref historique du corpus originel paru dans Baude et al. 2006 : 173-174, CLAPI s'est constitué sur une quarantaine d'années, à des fins de description de la langue parlée en interaction; la collection totalisait, en 2005, 600 heures d'enregistrements, en arrière-plan des 15 extraits longs (de plus de $2 \mathrm{~h}$ ) fournis au grand public en libre accès avec transcriptions et métadonnées. Puis, une quinzaine d'années après, CLAPI-FLE a été façonné, offrant une quarantaine de morceaux choisis souvent limités à moins de quatre minutes d'enregistrement audio ou vidéo ${ }^{5}$ comme conseillé pour les exercices d'écoute en classe de langue. La collecte des interactions pour constituer le CLAPI originel ne visait donc en rien le FLE, même si des collaborations avec de nombreux didacticiens du monde entier se sont tissées par la suite. Cet intérêt « tardif » a fait l'objet de précisions par les chercheurs de l'équipe de Lyon eux-mêmes, qui estiment que les développements en didactique des langues ont pleinement pris corps vers 2010 selon Baldauf-Quiliatre et al. $2016: 15$.

De même, le Corpus de Français Parlé Parisien des années 2000 (CFPP2000) se veut, à l'origine, une photographie des représentations de la ville de Paris, de ses quartiers et de ses banlieues, qui donne la parole à des Parisiens de souche ou plus récents, de générations différentes, sur plusieurs dizaines d'années, avec une motivation d'ordre sociolinguistique. En dehors des travaux de Branca-Rosoff, fidèles à cette orientation première, les principaux articles de recherche qui en découlent actuellement (listés sur le site dédié) produits par Lefeuvre, Pirès, ou Roig, portent principalement sur les caractéristiques syntaxiques et les corrélations que les modes d'interrogation de ce vaste corpus autorisent. Le site CFPP2000 n'a d'ailleurs toujours pas de volet d'applications en didactique des langues, même si les transcriptions et écoutes segmentées qu'on y trouve sont précieuses pour la classe de FLE et appréciées des enseignants auxquels on les présente. Enfin le corpus francophone collecté à l'échelle mondiale qu'est PFC est né du souci de décrire de manière exhaustive les variétés phonétiques et phonologiques du français parlé : ultérieurement, il a développé un riche ensemble d'exploitations pédagogiques, avec des ressources réalisées en partenariat avec le Centre de langues de l'université de Genève et tout un réseau de correspondants internationaux.

Au plan de l'histoire des disciplines, c'est donc une fois achevée (ou bien avancée) la description des collections de langue parlée que la grande majorité des auteurs de ces sites a pris conscience de l'intérêt de telles données pour la classe de FLE. Seul le site «France bienvenue, de vraies conversations pour apprendre le français comme on le parle et tout pour le comprendre " (https://francebienvenue1.wordpress.com/) part d'une intention didactique maintenue à toutes ses étapes de constitution; cependant il offre des échantillons d'interactions provoquées, sous forme de conversations entre étudiants de l'IUT de Toulon/Marseille, ce qui peut introduire des biais (bien que les échantillons fournis soient très « naturels »). 


\section{II.1.2 Des sites qui reflètent des enjeux et des positionnements disciplinaires complexes}

Le retour sur la généalogie des corpus d'interactions accessibles en ligne montre aussi et surtout depuis 2016 que nous sommes actuellement à un moment particulièrement délicat en matière d'interdisciplinarité : si les linguistes et sociolinguistes ont fait un pas en direction de l'enseignement des langues et du FLE en particulier, rien n'indique encore que les didacticiens s'approprient les corpus et les recherches en question ni y voient un apport majeur dans la mesure où la didactique des langues ne constitue plus depuis longtemps un champ d'application des descriptions linguistiques comme rappelé par Veronique 2009. "L'emprunt» mentionné dans le titre de notre contribution ne va pas de soi, alors même que les tentatives de transfert des résultats d'une discipline à l'autre viennent quasiment toutes des auteurs des collections numérisées, linguistes et sociolinguistes, associés à une poignée de chercheurs partenaires didacticiens qui testent et alimentent ces plateformes, à l'image de Ravazzolo et al. 2015 et de Detey et al. 2018. Le recours aux corpus numériques d'interactions en cours de FLE reste en pratique confidentiel et émerge dans la littérature académique de manière très récente, comme dans Calinon et al. 2019.

Pourtant, la didactisation progressive des collections d'interactions répond à une forte demande sur le terrain. Elle rencontre tout d'abord les principes de l'approche communicative encore très prégnante dans les pratiques d'enseignement pour le FLE, laquelle pousse à utiliser en cours des documents authentiques. Beaucoup de professeurs de langue en exercice ressentent de fait une grande frustration devant le peu de place accordée aux interactions sur le vif dans les manuels mais ces praticiens surchargés n'ont pas pour autant le courage d'aller puiser directement dans les ressources brutes que sont les émissions télévisuelles et les vidéos sur You Tube. La sélection et le montage des extraits authentiques sont en effet extrêmement chronophages, d'où la demande de données accessibles, archivées par thèmes, niveau de langue, actes de paroles, traits d'oralité, qui ont l'avantage d'être prêtes à l'emploi et gratuites tout en compensant les lacunes du matériel édité payant.

En dehors de ces raisons pratiques, la création d'exploitations didactiques par des chercheurs initialement attachés à l'analyse linguistique (et/ou discursive) peut revêtir un caractère plus politique, en lien avec les modes de légitimation actuels de l'activité de recherche. Le financement de la recherche requiert désormais de prouver l'utilité des travaux conduits. Le rapprochement entre linguistes, analystes du discours et didacticiens du FLE est somme toute en phase avec la nécessité d'une valorisation des connaissances produites. Mais en cela, les préoccupations didactiques des linguistes sont parfois perçues par les didacticiens comme une tentative de récupération de leur champ d'intervention. L'emprunt n'est donc pas toujours le bienvenu, d'autant que travailler à partir de data, c'est aussi mettre un pied dans le champ de la linguistique de corpus, qui fait la part belle à des problématiques d'apprentissage allant parfois à l'encontre de certains principes d'enseignement bien établis dans l'univers du FLE.

Boulton et Tyne 2014 montrent à ce propos comment l'exploitation de telles données par les didacticiens des langues a été initiée par les chercheurs anglophones mais aussi combien ces pratiques gagnent du terrain en France, en favorisant l'autoapprentissage, les apprentissages hybrides, l'accompagnement langagier. De telles évolutions ont certes le mérite de raccrocher le champ de la didactique du FLE, souvent 
replié sur lui-même, à la didactique d'autres langues, mais elles peuvent aussi être perçues comme des menaces par une didactique du FLE qui fait encore la part belle aux pratiques de classe à partir de supports sélectionnés par l'enseignant, un chef d'orchestre qui construit une progression de cours pensée à l'échelle du groupe.

Les aspirations des uns et des autres ne se rencontrent donc pas en tous points, même s'il est clair que mettre un pied dans des projets relevant des humanités numériques, actuellement très porteurs au plan international, est un atout de taille pour un chercheur francophone didacticien des langues. $\mathrm{Au} v \mathrm{vu}$ de ces intérêts tantôt concordants tantôt divergents, il apparaît que le vaste mouvement de mise à disposition de corpus numériques pré-pédagogisés ne peut être accueilli à bras ouverts ni accepté instantanément par les acteurs les plus établis de la didactique du FLE, ce qui explique notre choix d'en passer par la formation de jeunes professeurs sensibles aux questions pratiques, gagnés aux avantages du numérique et capables d'opérer des rapprochements disciplinaires sans trop de craintes ni d'arrières pensées. Nous pensons qu'à terme le recours régulier aux corpus d'interactions numérisées pour apprendre et enseigner une langue est très riche car ces corpus didactisés en ligne permettent, sans perdre temps, d'aller au contact d'interactions semblables à celles qu'on entend réellement, accessibles sous des formats aisément manipulables.

\section{II.2 Une diffusion sur le mode réduit de l'« échantillon »}

41 Si les sites mentionnés précédemment sont précieux pour les enseignants comme pour les étudiants, la présentation sur le mode d'extraits choisis de ces collections ainsi que l'analyse souvent très technique des caractéristiques interactionnelles qui y est menée par les chercheurs linguistes interrogent doublement le didacticien.

\section{II.2.1 Des morceaux choisis encore problématiques ?}

42 Comme l'ont remarqué bien plus vite que nous les mastérants de FLE, on n'échappe pas, dans les collections menées selon les principes de l'entretien sociolinguistique, à l'écueil déjà signalé pour les manuels, à savoir le lancement bref d'un thème par l'interviewer suivi d'une réponse longue sans grande alternance des tours de paroles. Ceci est par exemple flagrant dans Blanche-Benveniste $2002^{6}$, qui n'offre certes pas encore de site numérisé mais en préfigure la démarche grâce à un CDROM proposant l'écoute de 35 témoignages. Dans ce matériel, on a affaire à des prises de paroles introduites par une question, mais qui peuvent s'écouler ensuite durant de longues minutes de manière ininterrompue. Ceci se produit parfois aussi dans le Corpus de Français Parlé Parisien, où l'entretien semi directif est encore très structurant des échanges restitués, surtout durant la première période numérisée. Mais quand plusieurs personnes sont interrogées simultanément, ce biais s'estompe, à l'image de l'extrait avec Jeanne Mallet et Louise Liotard, de 2008.

Plus on avance dans le temps d'ailleurs plus un effort est fait pour que les corpus échappent au schéma rigide de l'entretien. Ainsi la deuxième partie du corpus orléanais ESLO 2 (dont l'élaboration est retracée par Baude et Dugua 2016) tente-t-elle d'instaurer des échanges à trois ou quatre personnes (comme les micro-trottoirs à la sortie de cinémas) ${ }^{7}$. De même, les plus récents enregistrements du Corpus de français parisien et la plupart des interactions proposées sur CLAPI-FLE portent une attention toute particulière aux polylogues (c'est-à-dire aux échanges alternant plus de deux 
interlocuteurs). Par cette volonté de présenter dans la quasi-totalité de ses extraits une parole émergente et souvent entrecoupée de chevauchements et de coupures, CLAPIFLE est d'ailleurs tout à fait exceptionnel par rapport à l'ensemble des autres corpus numériques.

\section{II.2.2 Les contraintes d'un accès rapide par mots clés}

Par-delà la tradition de l'entretien, la prédominance d'échanges faiblement alternés dans les pages dédiées aux exploitations pédagogiques des corpus oraux peut s'expliquer par le souci de donner à écouter des documents sonores "propres » et « représentatifs » de phénomènes pré-catégorisés en mots clés. Mais cela pourrait bien jouer dans le sens contraire de celui recherché et limiter l'exposition des étudiants au français tel qu'il est parlé.

Ainsi, les recherches les plus récentes sur les interactions montrent combien temporalité et co-construction du discours sont structurants en français oral, que l'on pense à la notion de "conversation mobile » ou de "grammaire dans l'interaction" mises en valeur par différents auteurs du numéro 175 de la revue Langue française faisant état de travaux récents, sensibles à la manière dont les échanges se structurent en temps réel. Dans cette publication collective, on retiendra en particulier la réflexion de Pekarek Doehler et Stoenica 2012: 121 qui déclarent s'interroger sur « la nature émergente, temporelle et interactionnellement contingente de ce qui est communément appelé "grammaire » mais aussi la conception prônée par Mondada 2012 : 144 d' « une grammaire émergente, imbriquée dans son écologie et dans les corps en interaction ». Un tournant épistémologique d'importance est donc à signaler dans la recherche contemporaine sur les interactions, en vertu duquel les caractéristiques de l'oral parviennent enfin à se dégager de la comparaison avec l'écrit et à ne plus être pensées comme des éléments déviants. Pekarek Doehler et Horlacher $2015: 141$ estiment que

«les structures dites non standard sont souvent l'aboutissement de réajustements complexes qui sont motivés par des besoins proprement interactionnels (et pas seulement grammaticaux) [...] les trajectoires syntaxiques des énonces s'agencent et se construisent au fur et à mesure de leur formulation ; le langage est un système

adaptatif, en émergence constante à travers son usage. ».

Malgré leur pertinence, de telles recherches ne filtrent pas dans les extraits de corpus numériques destinés aux enseignants de FLE. Selon nous, il existe actuellement un risque majeur de n'indiquer à l'exploitation que ce qui est jugé exploitable par le public enseignant non spécialiste des interactions. Cela est à la fois sain si l'on espère une large audience mais conduit aussi à renforcer une certaine vision du français oral - au détriment d'une autre. Ce risque est minimisé cependant par la mise à portée d'explications et d'indications sur les principales notions de l'analyse des interactions, que les sites de corpus numérisés opèrent en grande majorité à destination des nonspécialistes, avec les dangers que nous verrons plus bas cependant.

\section{II.3 La place encore anecdotique en FLE du numérique}

Le dernier point qui pose question quand on se penche sur les corpus numériques d'interactions est le choix quasi exclusif du mode électronique pour en diffuser des échantillons et pour étendre au monde des enseignants de FLE les propositions 
d'exploitation didactique des corpus mis à disposition. Le fait de construire une nouvelle fois des espaces physiquement distincts du matériel institutionnellement dominant (que sont encore l'ouvrage de formation de professeur et le manuel pour un cours de langue) n'aboutit-il pas au final à une marginalisation consentie?

\section{II.3.1 Une stratégie de diffusion risquée}

Alors que nous n'en avions pas perçu les limites dans un premier temps, la multiplication des mises à portée de la recherche sur les interactions ainsi que des exploitations didactiques pour le cours de FLE sous un format majoritairement numérique a été interrogée spontanément par les étudiants en master 2 à Paris Descartes. Exceptées les publications " papier » de Ravazzolo et al. 2016, de Detey et al. 2018, qui se sont faites chez des éditeurs connus respectivement dans le domaine de l'enseignement du français et dans celui de l'enseignement de l'anglais, la démarche de diffusion en direction des professeurs de langues a pris la voie électronique. Et encore ces rares initiatives de publication sur support papier concernent toutes la formation d'enseignants et non l'élaboration de matériel à usage direct en classe de langue, de type manuel ou auxiliaire pédagogique.

Cette stratégie du «tout numérique » se comprend néanmoins aisément : elle assure un accès quasi-instantané à une information de première main sur la recherche en linguistique de corpus et donne la liberté aux utilisateurs de choisir dans une gamme variée d'échantillons sans en passer par le veto des éditeurs. Le canal électronique préserve aussi la qualité du son et du geste, avec moins de déperdition que lorsqu'on en passe par un manuel papier ou un livre numérique, en particulier quand les extraits sont manipulés durant les cours. Cela étant, une telle mise en ligne pourrait paradoxalement constituer une limite, si elle ne s'accompagne pas simultanément d'une pénétration massive de l'ensemble du matériel didactique en FLE.

Si on observe les modes de diffusion des recherches et des données sur les interactions verbales en FLE, tout se passe en effet comme si d'un côté les maisons d'édition reconnues dans le domaine produisaient leurs ouvrages en saupoudrant dans les dialogues des manuels quelques traits d'oralité alors que de l'autre, les exemples d'interactions sur le vif ${ }^{8}$ sont réservés aux différents sites en ligne. À ce jeu-là, il se pourrait dans un proche avenir que la didactique des langues ne joue plus son rôle de discipline intégrative par rapport aux disciplines "mères » des Sciences du langage, lesquelles aménagent progressivement, à côté d'elle, des exploitations diverses de leurs résultats. D'où la question pour les chercheurs en analyse des interactions de réinvestir la production plus officielle de matériel pédagogique et les programmes éducatifs, afin que leurs résultats rayonnent dans les enseignements de FLE.

\section{II.3.2 Des outils pour la formation des enseignants ou au bénéfice des étudiants de langue?}

51 Sans doute conscients du danger de ne pas être pleinement compris ou suivis dans leur entreprise de mise à disposition de ressources pour le domaine du FLE, les auteurs de sites d'interactions numérisées ont quasiment tous adjoint à leurs collections des éléments propres à assurer eux-mêmes un début de formation des enseignants de FLE. Les introductions à l'analyse descriptive adressées aux enseignants de langue existent ainsi directement sur les sites de collections de corpus. 

interactions, l'enseignant de FLE va avoir tendance à réutiliser en cours une part du métalangage analytique spécialisé qu'il vient d'acquérir. Cette tendance est d'autant plus forte que les exploitations d'extraits d'interactions qui leur sont soumises consistent à déployer en cours des activités de description et de mise en lumière de caractéristiques de l'oral à partir d'échantillons d'interactions, d'une manière finalement symétrique à la propre analyse à laquelle ils ont été initiés peu de temps auparavant. Le risque de multiplier le métalangage en cours est donc loin d'être exclu, alors même que les méthodologies communicatives et actionnelles conseillent de limiter ce dernier. Par ailleurs, de tels éléments terminologiques ne peuvent pas être aisément maniés avec les apprenants étrangers de niveau inférieur à B2, qui forment le gros du public FLE au niveau mondial.

Une fois familiarisés avec les sites d'interactions prépédagogisées, en réalité, les étudiants futurs enseignants de FLE expriment le souci que l'apprentissage des catégories d'analyse auquel ils se prêtent soit rentable, opératoire et rende plus satisfaisant le travail de l'oral en cours. Dans ces conditions, on ne saurait faire l'économie d'une réflexion d'ensemble sur une exploitation efficiente des corpus d'interactions numérisées, qui diffuserait les résultats de l'analyse linguistique descriptive tout en renforçant l'exposition discursive des apprenants à un français oral ayant quelque crédibilité. 


\section{II.3.3 Vers une appropriation par les didacticiens} d'analyse dans des pratiques pédagogiques innovantes n'est pas une étape facile. Dans leur ouvrage consacré à la conception et à la mise à disposition du corpus PFC, Varieties of spoken french, Detey ${ }^{9}$ et al. 2016: 41 s'interrogent justement sur la manière de convertir des données phonologiques en ressources pédagogiques et proposent dans la partie « séquences pédagogiques » (pp 45-49) un dispositif pour conduire les étudiants à passer du code oral au code écrit et inversement. À partir de l'écoute d'extraits, un tableau suggère de pratiquer un résumé écrit, de fournir aux étudiants de FLE une transcription exacte mais aussi une version écrite plus accessible de la transcription dite " pedagogical oral discourse ", qui peut être remodelée en " discours écrit adapté " ("Adapted written discourse »). Ce "discours écrit adapté» constitue une forme intermédiaire expurgée pour l'écrit, comme on en trouve dans les entretiens produits dans la presse sur le mode direct. On franchit ainsi tous les degrés entre le "fresh talk » et ses restitutions possibles, ce qui implique une prise de conscience très fine des caractéristiques de l'oral et de l'écrit. Un développement de ces propositions didactiques se retrouve également dans la version en français de l'ouvrage en anglais, au chapitre 5 «Des données linguistiques à l'exploitation didactique " (Detey et Nouveau $2018: 107-137)$ avec des propositions de fiches pédagogiques dont celle p. 113 intitulée « De la compréhension détaillée à la réécriture ». Il est à noter enfin que le site Floral-PFC propose en ligne des ressources pédagogiques, en indiquant comment la simplification des échanges authentiques en vue de cours de français a été opérée (https://www.projet-pfc.net/le-projet-pfc-ef/ressources-didactiques/ sequencespedago/).

Sans reprendre à notre actif les transformations d'un type d'oral à l'autre et de l'oral à l'écrit proposées par Detey et al., nous avons été fortement marquée par l'idée de remodeler des échantillons authentiques, par petites touches. Cela nous a inspiré un dispositif de réécriture de dialogues préexistant dans le matériel édité, dispositif qui fait fructifier les principaux acquis de l'analyse des interactions mais n'impose pas de les nommer systématiquement face aux étudiants en cours de FLE. Cette stratégie de «retouche» des dialogues fabriqués édités est exposée dans la dernière partie du présent article.

\section{Travaux pratiques en formation d'enseignants de FLE}

L'un des principaux obstacles à l'utilisation des sites d'interactions prépédagogisées rencontrés par des enseignants débutants, comme le sont les étudiants du M2 de Paris Descartes, est le caractère chronophage de la sélection d'extraits pour préparer des séquences, y compris en puisant dans des bases de données "prêtes à l'emploi ". L'adaptation des propositions didactiques qui d'une certaine manière constituent une aide " prémâchée » prend en réalité beaucoup de temps, dans la mesure où les activités doivent faire sens par rapport à une progression d'ensemble, à un programme voire parfois aux séquences d'un manuel édité, imposé par l'employeur de ces enseignants comme fil rouge dans l'enchaînement des séances. Pour des enseignants de FLE peu 
avertis, proposer une solution alternative s'imposait. On a donc substitué aux préparations de séquences didactiques à partir d'échantillons d'interaction, pour l'un des deux devoirs notés dans le semestre, une tâche de réécriture de dialogue, optionnelle la première année puis rendue depuis 2018 obligatoire au vu de son intérêt formatif.

\section{III.1 La pratique de remodelages instructionnels}

Pour les travaux pratiques, nous avons sélectionné un dialogue de Quartier d'affaires 2, présentant la rencontre fortuite de deux anciens collègues (dont la retranscription au kilomètre figurait à la fin de l'ouvrage et se trouve reproduite en Annexe 3). Ce dialogue, donné à écouter également en ligne sur la plate-forme Moodle, présente une certaine spontanéité et de nombreux marqueurs de structuration du discours (interjections, marqueurs phatiques), ce qui a guidé notre choix. Cela étant, il tourne vite également à l'enchaînement question/réponse avec des éléments d'explication longue de l'activité professionnelle. Aussi a-t-on a invité les étudiants à «rendre l'échange plus naturel et conforme à la dynamique interactionnelle du français ».

Une liste de modifications à répartir librement dans le dialogue fabriqué (dit « dialogue souche ») a été distribuée, en prenant soin que chaque modification soit codée visuellement (par des polices et des couleurs différentes) en fonction de la nature de la retouche apportée (exprimée au moyen de la terminologie propre à l'analyse interactionnelle, déjà étudiée en cours). Comme on opérait à partir d'un matériel de spécialité, on a également poussé les étudiants à réinvestir les acquis de l'analyse des discours et des actions en contexte professionnel.

Une première série d'instructions portait ainsi sur la précision et la modification des éléments physiques constituant le cadre dans lequel l'interaction s'inscrit. Il a ainsi été demandé comme toute première instruction d' "Ajouter un participant de plus, qui vient se mêler à l'échange, soit sur le lieu de la rencontre, soit par téléphone. ». Parmi les scénarios imaginés par les étudiants on citera pour illustrer cette première retouche: l'arrivée d'une ancienne collègue de Stéphane et Gaspard, qui donne son point de vue sur la brochure promotionnelle de l'entreprise fondée par Stéphane (Nanty 2018); une cliente de Gaspard au téléphone, qui s'inquiète d'une commande (Laggoune 2018). Enfin on trouve l'appel téléphonique de l'épouse et collaboratrice de Stéphane (Clerc 2018), inséré dans l'échange de manière incidente [ajout mis entre crochets ci-après] :

Stéphane : Avec ma femme et un ami, on a monté une petite entreprise. Une start-

up!

[Le téléphone de Stéphane sonne

Stéphane : Ah bah tiens! Quand on parle du loup! C'est ma femme, tu m'en veux pas?

Gaspard : Non non bien sûr je t'en pr...

Stéphane : Allô, oui, dis-moi que tout se passe bien. OK. OK. Non non pas besoin. Je lui envoie moi. OK oui on fait ça. Je te laisse je suis avec Gaspard. Ah oui pourquoi pas, ben oui je lui propose. Allez à toute à l'heure. Non j'oublie pas promis. Excusemoi hein c'est ma femme mais c'est aussi le boulot (rires).]

La deuxième instruction concourrait également à renforcer l'ancrage matériel et à contextualiser l'échange plus fortement. On a ainsi demandé de « Faire apparaître des objets qui prendront leur place dans la conversation même ». Les objets qui ont surgi 
dans les propositions des étudiants ont été entre autres : un briquet (Ferragu 2018), un téléphone (Baron 2018), une carte professionnelle (Mougin 2018). Certains d'entre eux (comme une bouteille de lait) ont fait l'objet par les étudiants eux-mêmes d'une critique, au moment de la mise en commun, car ils ne relevaient pas du monde professionnel attendu dans de tels échanges et étaient donc peu plausibles ou représentatifs.

Il faut comprendre également que ces deux premières instructions ne précisent pas l'ordre d'apparition des modifications dans la chronologie du dialogue. Elles occasionnent donc des métamorphoses du texte souche très différentes selon les étudiants, ce qui montre la richesse des points d'arrimage d'une interaction à son contexte supposé.

Avec la troisième instruction, on entrait dans les modifications de détail des répliques déjà fournies dans le manuel, avec des exigences beaucoup plus techniques. Il a été demandé d' "étoffer la séquence d'ouverture en matière de salutations (et de surligner l'ensemble retouché en jaune). Le quatrième point consistait à « Faire apparaître une demande de précision suite à une incompréhension, par le biais d'une réparation hétéro-initiée de la part de Gaspard (à surligner en gris). Notre cinquième demande invitait à "opérer une dislocation à gauche pour introduire une réponse qui ne correspond pas aux attentes d'un des interlocuteurs, avec un «mais» pour introduire un autre point de vue ou une autre opinion. » Il fallait utiliser le souligné pour cette modification déclinée entre autres comme suit (Castro-Munoz 2018) :

Stéphane : On loue des villas, c'est plus classique, et toutes sortes d'objets... [Ça, les objets, ça marche super bien!

Gaspard : Mais les objets, ça se loue pas... moi je loue jamais d'objet ! ]

$\mathrm{Au}$ plan pédagogique, ce type de programme instructionnel est un peu une « heure de vérité ». On voit très clairement en lisant les propositions des étudiants ce qu'ils se sont appropriés du cours et les parties qui n'ont pas été comprises. Parfois la lecture d'un extrait plutôt technique (comme Traverso 2016: 44-45 sur les tours simples ou segmentés) a guidé l'ajout d'une instruction spécifique (de type "dans une réplique, ajoutez un tour de plus, que vous soulignerez). On voulait voir par cette instruction si les étudiants avaient bien cerné la notion de tour dans toute sa subtilité. De même, la lecture d'un article d'Oursel 2012 sur l'atténuation des refus a conduit à solliciter les étudiants en ce sens, dans certains autres exercices de remodelage les années précédentes.

67 De manière générale, les remodelages obtenus ont été très probants. Beaucoup de mastérants se sont révélés aptes à opérer les transformations requises dans des temps très limités, se limitant parfois à un atelier non noté d'une heure, en salle informatique. Nous avons pratiqué ce type de remodelage instructionnel trois ans de suite, avec des dialogues souches différents, souvent beaucoup plus brefs et simples que celui de Quartier d'Affaires 2. On livre Annexe 4 un extrait d'Objectif Diplomatie 1 2010: 10 qui a également été utilisé. Les étudiants en train de se former à devenir enseignants ont affiché une grande satisfaction, surtout à l'occasion des remodelages de dialogues brefs, à pouvoir ainsi produire rapidement et tout seuls des dialogues « vivants et crédibles ». Peut-être ont-ils vécu ces remodelages comme une sorte de customisation du matériel pédagogique?

68 L'étape ultime serait à présent de tester en cours de FLE ces dialogues « améliorés ». Si leur fabrication a rassuré les futurs enseignants, sont-ils vraiment exploitables et 
surtout profitables aux étudiants de FLE? Quoi qu'il en soit, l'emprunt des catégories d'analyse interactionnelle ne sera pas resté sans effet. Rendant les dialogues existants quasi insupportables aux étudiants, ces derniers sont passés du stade d'utilisateurs à celui de concepteurs innovants. Mais leur niveau d'exigence n'en a été que relevé.

\section{III.2 De quelques effets induits dans les manières d'enseigner}

69 Devenus auteurs, les étudiants ont perçu que quand ils dispensaient des cours de FLE, ils créaient aussi d'une certaine manière de la matière verbale, plus ou moins contrôlée. Comme on les avait sensibilisés aux recherches récentes sur les interactions professorales (dont Cicurel 2011), ils se sont mis à interroger leurs propres prises de parole en tant qu'enseignants. Que produisaient-ils au juste comme échanges?

70 Cette interrogation a pris selon les étudiants une ampleur variable. Certains se sont contentés d'interroger la répartition de la parole qu'ils opéraient lors des pratiques de classe imposées par le stage pédagogique de master. Pour d'autres, le questionnement a revêtu la forme d'une "mise en miroir» tous azimuts, comme Pych 2019, qui a remarqué suite aux cours sur les interactions, qu'il parlait à ses propres apprenants de FLE sur deux registres : l'un très proche de l'oral spontané, aux marges des cours, dans les couloirs ou lors de questions auto-initiées, inattendues, ouvrant sur des réponses plus improvisées de la part de l'enseignant ; l'autre registre étant celui des explications préparées à l'avance, distillées en cours sur un ton plus docte, proches de l'écrit oralisé. Ce faisant, Pych s'est demandé si le français oral auquel un enseignant de FLE exposait ses étudiants ne constituait pas une forme de pratique interactionnelle très contrôlée, plus continue syntaxiquement que le français oral quotidien. On a pu réaliser alors qu'un enseignant qui a le français pour langue maternelle et qui exerce à l'étranger, n'offre pas toujours à ses étudiants une exposition à la conversation orale courante quand il échange avec eux : il peut en effet ne s'exprimer que sur le mode professoral ! D'où l'idée venue à certains mastérants de modifier leur propre discours durant les cours de langue qu'ils dispensaient, pour renvoyer ponctuellement dans leurs interactions avec les étudiants à du français parlé plus authentique.

\section{Conclusion}

71 A travers le récit d'une expérience d'enseignement de l'analyse des interactions à de futurs enseignants de FLE se dessine au final une triple interrogation. Se pose tout d'abord la question de l'acceptabilité des résultats de recherche d'une discipline par une autre, avec les enjeux de territoire complexes que nous n'avons fait qu'esquisser. Intervient aussi un questionnement sur les conditions de l'adaptation pédagogique d'éléments théoriques par le milieu éditorial comme par le professeur de FLE «lambda». Enfin, et ce n'est pas le moins important, le parcours que nous avons fait ces six dernières années n'a pas fait que bousculer les seuls mastérants dans leurs certitudes. Bien plus qu'à nos débuts, nous nous posons à présent la question lancinante de ce que vise l'enseignement du français oral en cours de langue. Plus nous avons avancé dans la réflexion sur la transposition des résultats de l'analyse conversationnelle et interactionnelle, plus nous nous sommes en effet rendu compte que les écueils rencontrés par les enseignants de FLE débutants recouvraient une somme de questions rarement explicitées. Vise-t-on réellement à exposer les 
apprenants à du français tel qu'il est parlé lorsqu'on pratique l'oral en cours de FLE? Ne serait-ce pas exiger de non natifs de parler comme des natifs? Les étudiants, qui ne viendront pas tous en France ou dans un pays francophone, ont-ils besoin de se conformer à ce qui est effectivement dit en français langue maternelle? Le travail de l'oral est-il là pour donner à comprendre ou donner à entendre? Ou alors pour permettre de s'exprimer comme l'on peut sur ce que l'on veut?

\section{Annexes}

\section{Manuels et auxiliaires pédagogiques étudiés (Classés par ordre alphabétique)}

Adomania A1/A2, 2016, Hachette.

A la Une, au cœur du monde francophone, 2019, Maison des Langues.

A l'écoute des Français, 1991, CLE International.

A l'écoute des professionnels, 1994, CLE International.

Alter Ego +, A2, 2012, Hachette FLE.

Campus 2, 2002, CLE International.

Clés pour l'oral - Manuel d'exploitation, 2011, Hachette FLE.

Edito B1, 2012, Didier.

En cuisine, A1/A2, 2014, CLE International.*

Français.com., 2002, CLE International.

Français.com, intermédiaire, (2011), CLE International.

Latitudes 3, B1., 2010, Didier.

Le français en contexte tourisme, A1/A2, 2013, Maison des langues.*

Objectif diplomatie 1, 2010, Hachette.*

Paroles en situations, 2015, Focus, Hachette.

Quartier d'affaires A2, 2013, CLE International.*

Reflets 2, 2000, Hachette.

Texto 1, 2016, Hachette.

\section{Sites de corpus oraux analysés}

CLAPI, Corpus de LAngue Parlée en Interaction, http://clapi.icar.cnrs.fr/

CLAPI-FLE, Corpus de LAngue Parlée en Interaction pour le FLE, http://clapi.icar.cnrs.fr/

FLE

COCOON, Collection de Corpus Oraux Numériques, https://cocoon.huma-num.fr/ exist/crdo/

CFPP2000, Corpus de Français Parlé Parisien 2000, Discours sur la ville, http://

cfpp2000.univ-paris3.fr/

ESLO, Enquêtes SocioLinguistiques à Orléans, http://eslo.huma-num.fr/

FRANCE BIENVENUE, de vraies conversations pour apprendre le français comme on le parle et tout pour le comprendre, https://francebienvenue1.wordpress.com/

ORFEO, Corpus d'étude pour le français contemporain, https://www.projet-orfeo.fr

PFC. (2015). FLORAL, Phonologie du Français Contemporain (PFC), Usages, variétés, structure, https://www.projet-pfc.net/

\section{Dialogue souche du manuel Quartier d'affaires 2, Unité 9, p. 120, soumis à modification}

Gaspard: Bonjour Stéphane! Comment tu vas? Tu travailles toujours chez Provita?

Stéphane : Eh bien non Gaspard! L'année dernière mon entreprise a fermé. J'ai donc dû chercher un nouvel emploi.

Gaspard : Tu as eu du mal à trouver du travail ?

Stéphane : Oui et non! J'ai été embauché dans une entreprise de produits informatiques, mais je ne suis pas resté. Les conditions n'étaient vraiment pas bonnes... 
Gaspard : Et alors?

Stéphane : Avec ma femme et un ami, on a monté une petite entreprise. Une startup!

Gaspard : Ah bon? Et vous faîtes quoi?

Stéphane : Nous louons toutes sortes de choses. En fait, nous faisons l'intermédiaire entre celui qui a quelque chose à louer, et celui qui doit louer quelque chose.

Gaspard : Je n'aurais jamais pensé à ça. Et ça marche?

Stéphane : Oui, parce qu'on propose des choses très diverses! Par exemple, on peut louer la cave ou le grenier de quelqu'un qui ne l'utilise pas. On est aussi en relation des entreprises, surtout des PMI, qui n'utilisent pas tout leur espace. On loue des villas, c'est plus classique, et toutes sortes d'objets...

Gaspard : Par exemple?

Stéphane : Oh, la liste est longue, ça va de l'ordinateur, aux costumes, en passant par la tondeuse à gazon, une caméra professionnelle, un sac à main... Une fois, on a même loué une trompette pour une journée! On a plus de 150000 objets en location, alors tu penses bien qu'il y a des trucs insolites!

Gaspard : Eh bien, justement, j'ai besoin d'une perceuse. Tu as ça?

Stéphane : Évidemment

Dialogue du manuel Objectif diplomatie, Unité 1, p. 10, soumis à modification

A l'accueil

Agent d'accueil : Bonjour Madame.

Marion Delorme : Bonjour Monsieur.

Agent d'accueil : Oui, vous êtes Madame...?

Marion Delorme : Je m'appelle Delorme, Marion Delorme, D.E.L.O.R.M.E., délégation

française

Agent d'accueil : Vous avez une pièce d'identité, s'il vous plaît?

Marion Delorme : Un passeport, ça va?

Agent d'accueil : Oui, très bien. Voici le badge. La salle de conférences est au niveau quatre.

Marion Delorme : Merci, au revoir !

Agent d'accueil : Au revoir Madame et bonne journée !

\section{BIBLIOGRAPHIE}

BALDAUF-QUILLIATRE, I. et al., 2016, « CLAPI, une base de données multimodale pour la parole en interaction : apports et dilemmes ", Corpus 15, http://journals.openedition.org/corpus/2991.

BAUDE, O., DUGUA, C., 2016, « Les ESLO, du portrait sonore au paysage digital », Corpus15, consulté le 30 avril 2019.

BAUDE, O. et al., 2006, Corpus oraux, guide des bonnes pratiques 2006, Paris et Orléans : CNRS Editions et Presses Universitaires d'Orléans.

BLANCHE-BENVENISTE, C., 2002, Choix de textes en français parlé- 35 extraits, Paris : Honoré Champion.

BOULTON, A., TYNE, H., 2014, Des documents authentiques aux corpus : démarches pour l'apprentissage des langues, Paris : Didier. 
CALINON, A.-S., HAMMA, B., PLOOG, K., SKROVEC, M., 2019, Linguistique interactionnelle, grammaire de l'oral et didactique du français, Besançon : Presses Universitaires de Franche Comté.

CICUREL, F., 2011, Les interactions dans l'enseignement des langues. Agir professoral et pratiques de classe, Paris : Didier.

DETEY, S., 2017, « Du statut de l'authenticité dans l'usage de corpus oraux pour la didactique du FLE : réflexions. Projet LLE-RECIF ", Communication orale à l'atelier "Comment concevoir une collection d'usages langagiers utile pour l'enseignement du FLE ?», Paris : Université Paris Descartes.

DETEY, S. et al., 2016, Varieties of spoken French, Oxford: Oxford University Press.

DETEY, S. et al., 2018, Les variétés du français parlé dans l'espace francophone, Ressources pour l'enseignement, Paris : Ophrys.

DEVELOTTE, C., 1996, « Les interactions discursives en jeu dans un système éducatif » dans Moirand, D. (Ed.), Le discours : enjeux et perspectives, Paris : Hachette, p. 142-149.

KERBRAT-ORECCHIONI, C., 2011, Le discours en interaction, Paris : Armand Colin.

LEBRE-PEYTARD, M., LIEUTAUD, S., BEACCO, J.-C., MALANDAIN, J.-L., 1977, Le document oral brut dans la classe de français, La transcription de documents sonores authentiques (Fascicule du BELC), Sèvres : CIEP.

LEBRE-PEYTARD, M., MALANDAIN, J.-L., 1982, Décrire et découper la parole, Sèvres : CIEP.

MONDADA, L., 2012, « Organisation multimodale de la parole-en-interaction : pratiques incarnées d'introduction des référents", dans V. Traverso (éd.), Analyses de l'interaction et linguistique : état actuel des recherches en français. Langue française 175, Paris : Armand Colin, p. 129-147.

MOURLHON-DALLIES, F., 2018a, «Enseignement du français aux publics professionnels et analyse des interactions au travail : un rendez-vous manqué ? ", dans L. Filliettaz, M. Laforest, I. Vinatier (éds), L'analyse des interactions dans le travail. Outil de formation professionnelle et de recherche Dijon : Éditions Raison et Passions, p. 127-149.

MOURLHON-DALLIES, F., 2018b, « Interactions en milieu professionnel : quelle voix prendre ?», Le français dans le monde 415, Paris : FIPF et CLE International, p. 24-25.

OURSEL, E., 2012, «Le refus de service dans les administrations : quelles formes d'atténuation pour ces actes menaçants et quelles offres de compensation ? ", Signes, Discours et Sociétés $n^{\circ} 9$, Université Galatasaray, http://www.revue-signes.info/document.php?id=2806.

PEKAREK DOEHLER, S. et STOENICA, I.-M., 2012, « Emergence, temporalité et grammaire-dansl'interaction : disloquée à gauche et nominativus pendens en français contemporain ", Langue Française 175, Paris : Armand Colin, p. 111-127. https://www.cairn.inforevue-languefrancaise-2012-3-page-111.htm

PEKAREK DOEHLER, S., HORLACHER, A.-S., 2015, « Grammaire émergente, grammaire pour l'interaction. Variations sur les constructions disloquées », dans X. Gradoux (éd.), Agir dans la diversité des langues, Louvain-la-Neuve : De Boeck, p. 139-151.

PORQUIER, R., 2001. « Mots-phrases, phrasillons, locutions-énoncés : aux frontières de la grammaire et du lexique en français langue étrangère ", Langue Française n¹01, p. 106-123.

PYCH, P., 2019, L'interaction spontanée en classe de FLE, Mémoire de master 2 DDL, Paris : Université René Descartes.

RAVAZZOLO, E., JOUIN, E., TRAVERSO, V., VIGNER, G., 2015, Interactions, dialogues, conversations : l'oral en français langue étrangère, Paris : Hachette FLE. 
RIVENC, P., 2006, «Les auteurs du Français fondamental face à un objet nouveau et insolite : l'interaction orale ", Documents pour l'histoire du français langue étrangère et seconde 36, Paris : SIHFLES. http://dhfles.revues.org/1185.

SANDRE, M., 2013, Analyser les discours oraux, Paris : Armand Colin.

TRAVERSO, V., 2007, « Pratiques communicatives en situation : objets et méthodes de l'analyse d'interactions ", Recherches en soins infirmiers 89, p. 21-33.

TRAVERSO, V., 2011 (réédition de 2005), L'analyse des conversations, Paris : Armand Colin.

TRAVERSO, V., 2012, Analyses de l'interaction et linguistique : état actuel des recherches en français. Langue française 175, Paris : Armand Colin.

TRAVERSO, V., 2016, Décrire le français parlé en interaction, Paris : Ophrys.

VERONIQUE, D., 2009, « La linguistique appliquée et la didactique des langues et des cultures : une polémique française au cœur d'un débat international ", Le Français dans le Monde Recherches et applications 46, Paris : FIPF et CLE International, p. 42-52.

WEBER, C., 2006, « Pourquoi les Français ne parlent-ils pas comme je l'ai appris ? », Le français dans le monde 345, Paris : FIPF et CLE International, p. 31-33.

WEBER, C., 2013, Pour une didactique de l'oralité - Enseigner le français tel qu'il est parlé, Paris : Didier.

\section{NOTES}

1. Par interaction " réaliste ", on entend un document fabriqué qui reconstitue et concentre les particularités des interactions authentiques mais ne correspond à aucune d'elles exactement.

2. Ce choc est d'autant plus grand que près de la moitié des étudiants a suivi en M1 un cours optionnel d'analyse conversationnelle et interactionnelle et que toute la cohorte de M1 a reçu également un enseignement de «syntaxe du français et FLE » qui donne les outils d'analyse du français oral en interaction, entrées également récapitulées lors des deux premiers cours du M2 que nous animons.

3. Nous avons notamment mis en lumière que les interactions professionnelles étaient très souvent centrées sur une tâche problème mettant en débat des choix d'action; qu'elles donnent tour à tour la parole à au moins deux protagonistes, avec des incidences fréquentes (appels téléphoniques, irruption d'un protagoniste supplémentaire) ; qu'elles supposent souvent la manipulation d'objets. En outre, il ne s'agit pas de discours sur le travail mais de discours soutenant un travail en cours.

4. Calinon et al. (2019) parviennent à la même conclusion, en particulier dans la contribution de Pires sur l'usage de «nous » et de « on » dans les manuels pour débutants puis dans le chapitre rédigé par Hamma (sur le passif tel qu'il est présenté dans le matériel édité).

5. Ces trois dernières années, deux étudiantes mastérantes de Paris Descartes ont d'ailleurs participé à la sélection de ces micro extraits et à leur didactisation, à savoir Sabine Hervé et Martina Ronci, dans le cadre de leur stage de M2. Il faut aussi mentionner l'importante contribution d'E. Oursel et des mastérants de FLE de Paris 8 dans la constitution des fiches explicatives et des séquences pédagogiques.

6. On ne peut pas reprocher vivement ce caractère peu dialogué à l'auteur en question car sa perspective, à l'époque était plus syntaxique qu'interactionnelle. Mais le mode de découpe et de partage des entretiens enregistrés est typique de ce qui suivra.

7. Pour plus de précision sur la constitution en deux grandes périodes du corpus ESLO, nous renvoyons à http://eslo.huma-num.fr/index.php/pagepresentation/pageeslopresentation 
8. Nous employons l'expression « interactions sur le vif » afin d'éviter ici des qualificatifs du type " authentiques", « réelles", « effectives» c'est-à-dire pour bien marquer la dynamique interactionnelle à valoriser dans les corpus oraux, dynamique structurante que nous estimons très importante. Il faut noter dans le présent numéro de Linx que Ravazzolo et Etienne parlent de « corpus écologiques d'interactions », pour bien signifier la nature multimodale et l'extraction de leur environnement naturel sans modification des échantillons qui les occupent. La désignation des interactions est donc une manière d'insister, pour les besoins de son propre propos, sur telle ou telle caractéristique des corpus didactisés. On peut remarquer au passage qu'il n'existe pas d'appellation stabilisée (on parle de «français parlé», de "français entendu», d' « interactions ») ce qui montre que ces corpus sont au carrefour de préoccupations multiples.

9. Nous attirons en particulier l'attention sur cet auteur, qui a également développé une réflexion de ce type en 2017 lors d'une communication orale articulée autour de la notion d'authenticité dans une journée scientifique à Paris Descartes référencée en bibliographie.

\section{RÉSUMÉS}

Depuis peu, l'analyse des interactions a mis à la portée des enseignants de FLE ses principaux résultats de recherche (Ravazzolo et al. 2015, Traverso 2016, Detey et al. 2018, Calinon et al. 2019) tout comme des corpus audios et vidéos pré-didactisés en libre accès (CLAPI-FLE, PFC). En parallèle, la classe et les échanges qui s'y tiennent ont été placés au centre de l'analyse des interactions didactiques (Cicurel 2011). Mais tous ces croisements disciplinaires n'ont pas été neutres. En sensibilisant les futurs enseignants de FLE à l'analyse interactionnelle, on constate que ces derniers posent à présent des questions que leurs ainés n'ont pas résolues : pourquoi le matériel édité est-il aujourd'hui encore porteur de dialogues fabriqués, très éloignés de ce qu'on entend? Que faire des interactions tenues par l'enseignant à la marge des cours avec les apprenants? Que penser de l'exposition discursive que réalise en cours l'enseignant par sa propre parole ? Autant d'interrogations qui heurtent des pratiques didactiques très largement dominantes, lesquelles posent le document comme support, le cours comme unité et le professeur comme chef d'orchestre.

Recently, analysis of interactions has put its main research results (Ravazzolo et al. 2015, Traverso 2016, Detey et al. 2018, Calinon et al. 2019) as well as audio and video corpora prepped for instruction (CLAPI-FLE, PFC) within the reach of French as a foreign language teachers. In parallel to that, the classroom and the conversations taking place therein have been the center of attention of pedagogical interaction analysis (Cicurel 2011). But these disciplinary criss-crossings have not been unimpacting. In sensibilizing the future teachers of French as a foreign language to the analysis of interactions, we find that these students are beginning to ask questions that their elders have not answered yet: why does the pedagogical material published nowadays still include dialogues that are so far apart from authentic conversations? What as to be done with conversations that the teachers and learners have aside from the course itself? What can we make of the discursive input given by the teacher in the classroom, with their own speech? Many questions that confront the largely dominating practices, where the document stands as teaching material, the course is a unit and the teacher is the orchestrator. 
INDEX

Mots-clés : français parlé ; manuels de langue ; analyse interactionelle, corpus oraux

Keywords : spoken French ; language textbooks ; interactional analysis, oral corpora

\section{AUTEUR}

\section{FLORENCE MOURLHON-DALLIES}

Laboratoire EDA, Université Paris Descartes 